\title{
Controle robusto de horizonte finito em sistemas lineares com parâmetros variantes no tempo e saltos Markovianos
}

\author{
Rosileide de Oliveira Lopes* Eduardo M. A. M. Mendes ** \\ Alessandro N. Vargas ${ }^{* * *}$ Reinaldo M. Palhares ${ }^{* *}$ \\ * Instituto de Ciências Puras e Aplicadas, Departamento, Universidade \\ Federal de Itajubá, MG, (e-mail: rosileidelopes@unifei.edu.br). \\ ** Departamento de Engenharia Eletrônica, Universidade Federal de \\ Minas Gerais, MG, (e-mails: emmendes@ufmg.br, rpalhares@ufmg.br) \\ *** Departamento de Engenharia Elétrica, Universidade Tecnológica \\ Federal do Paraná, PR, (e-mail: avargas@utfpr.edu.br)
}

\begin{abstract}
This paper presents a strategy to calculate a suboptimal solution to the finite-time linear-quadratic control problem of Markov jump linear systems. When the mode is fixed, the system has parameter-varying matrices that take values in a polutopic set. An upper bound for the cost function is obtained and, as a consequence, the feedback gains are calculated by solving an optimization problem written in terms of linear matrix inequalities. A numerical example is given to illustrate the effectiveness of the proposed method.

Resumo: Este trabalho apresenta uma estratégia para calcular uma solução subótima para o problema de controle linear quadrático de tempo finito de sistemas lineares com saltos Markovianos. Quando os modos são fixados, o sistema tem matrizes com parâmetros variantes, os quais tomam valores em um conjunto politópico. Um limitante superior para a função custo é obtido, como consequência, os ganhos de realimentação são calculados resolvendo um problema de otimização escrito em termos de desigualdades matriciais lineares. Um exemplo numérico é dado para ilustrar a eficácia do método proposto.
\end{abstract}

Keywords: robust control; finite-time; linear systems; Markov jump.

Palavras-chaves: controle robusto; tempo finito; sistemas lineares; saltos Markovianos.

\section{INTRODUÇÃO}

Este artigo considera um problema de controle ótimo de tempo finito de uma classe específica de sistemas estocásticos em tempo discreto, nomeadamente sistemas lineares com parâmetros variantes no tempo, saltos Markovianos (SLPVSM) e ruído aditivo.

Os sistemas lineares com saltos Markovianos podem ser encontrados em muitas aplicações reais, como sistemas econômicos, sistemas de energia, sistemas de comunicação, sistemas químicos, sistemas de controle em rede, sistemas de manufatura e sistemas de controle (Shi and Li, 2015). Tais sistemas são usados para modelar processos que apresentam mudanças abruptas em sua estrutura, que podem ser o resultado de falhas de componentes ou reparos, distúrbios do meio, mudanças abruptas no ponto de operação, etc. (Costa et al., 2006). Muitos pesquisadores têm abordado questões relevantes sobre tais sistemas, como estabilidade, filtragem, síntese de controle e controle ótimo, etc. ((Shi and Li, 2015), (Shi et al., 1999), (Costa et al., 1999), (Vargas et al., 2013), (Geromel et al., 2009), (Morais et al., 2018), (Costa and Tuesta, 2003), (Oliveira et al., 2014)).

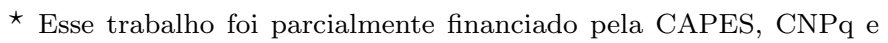
FAPEMIG.
Dentro do conjunto de sistemas que podem ser representados como sistemas de saltos Markovianos, podem também ser considerados sistemas em que as matrizes apresentam variações (politópicas) de parâmetros associadas com os modos da cadeia de Markov, formando assim os sistemas com parâmetros lineares variantes com saltos Markovianos SLVPSM, veja, por exemplo, (Gagliardi et al., 2012; Kim, 2017; Lopes et al., 2019; Ren and Zong, 2017; Zhu et al., 2017).

Trabalhos no contexto de variação de parâmetros com saltos Markovianos podem ser encontrados em filtragem (Gagliardi et al., 2012), (Zhu et al., 2017) e (Ren and Zong, 2017), para controle preditivo robusto baseado em modelo (Lopes et al., 2019) e para estabilização (Kim, 2017).

Há interesse em resultados sobre controle para sistemas lineares com saltos Markovianos considerando horizonte infinito, veja, por exemplo, (Mariton, 1990; Costa et al., 2006; Do Val et al., 2002; Costa et al., 2014). Esse trabalho no entanto, se concentra em controle de horizonte finito.

A principal contribuição deste artigo é encontrar uma solução subótima para o problema de otimização linear quadrático de horizonte finito para sistemas SLPVSM discretos no tempo com um termo de ruído aditivo. A abor- 
dagem é empregada por meio de desigualdades matriciais lineares.

O artigo está organizado como segue: Na Seção 2 algumas notações e a formulação do problema são apresentados. Resultados preliminares e o resultado principal são expostos na Seção 3 e 4, respectivamente. Uma simulação numérica é dada na Seção 5. Finalmente, a Conclusão é apresentada na Seção 6.

\section{FORMULAÇÃO DO PROBLEMA}

A notação empregada neste artigo é como segue. $\mathbb{R}_{+}$ representa o conjunto dos números reais positivos. $\mathbb{R}^{n}$ representa o espaço Euclidiano $n$ dimensional e $\mathbb{R}^{n \times m}$ representa o espaço linear normado de todas as matrizes reais $n \times m$. Denota-se por $\mathcal{S}^{n}$ o subespaço linear normado de $\mathbb{R}^{n \times n}$ de matrizes simétricas $\left\{U \in \mathbb{R}^{n \times n}: U=U^{\prime}\right\}$, no qual $U^{\prime}$ denota a transposta da matriz $U$. Considere $\mathcal{S}^{n 0}$ $\left(\mathcal{S}^{n+}\right)$ como o cone convexo fechado (aberto) de matrizes positivas semidefinidas (definidas) $\left\{U \in \mathcal{S}^{n}: U \geq 0\right.$ (> $0)\} . \Theta=\left\{1, \ldots, n_{\theta}\right\}$ indica um conjunto finito e $\mathbb{S}^{n+}$ representa o conjunto formado por um número $n_{\theta}$ de matrizes tais que $\mathbb{S}^{n+}=\left\{U=\left(U_{1}, \ldots, U_{n_{\theta}}\right): U_{i} \in\right.$ $\left.\mathcal{S}^{n+}, i \in \Theta\right\} . \mathbb{E}[v]$ denota o valor esperado da variável aleatória $v$. \|. || representa a norma euclidiana. $\mathcal{I}$ representa a matriz identidade com dimensões apropriadas.

Considere o espaço de probabilidade completo $\left(\Omega ;\left(\mathfrak{F}_{n}\right)\right.$; $\mathfrak{F} ; P)$ com filtragem $\left\{\mathfrak{F}_{n}, n \geq 0\right\}$ que atende as condições normais (seção 2.3, [Costa et al., 2006]), associado ao sistema linear com saltos Markovianos:

$$
\left\{\begin{array}{l}
x_{n+1}=A_{\xi_{n}}\left(\theta_{n}\right) x_{n}+B_{\xi_{n}}\left(\theta_{n}\right) u_{n}+E\left(\theta_{n}\right) w_{n} \\
y_{n}=C\left(\theta_{n}\right) x_{n}+D\left(\theta_{n}\right) u_{n}, n \geq 0
\end{array}\right.
$$

$x_{n} \in \mathbb{R}^{n_{x}}, u_{n} \in \mathbb{R}^{n_{u}}, w_{n} \in \mathbb{R}^{n_{w}}$ denotam os estados do sistema, as entradas de controle e o ruído aditivo do sistema, respectivamente. O estado inicial $x_{0}$ e o modo inicial $\theta_{0}$ são dados. $O$ processo $\left\{\theta_{n} ; n=0, \ldots\right\}$ representa uma cadeia de Markov discreta no tempo tomando valores no conjunto $\Theta$ com matriz de probabilidade de transição $\mathbb{P}=[p(i, j)]_{i, j \in \Theta} \operatorname{com} p(i, j)=\operatorname{Pr}\left[\theta(n+1)=j \mid \theta_{n}=\right.$ $i]$. Assume-se que o ruído do processo $\left\{w_{n} ; n \geq 0\right\}$ é composto de vetores aleatórios mutuamente independentes com média zero, covariância igual a $\Sigma$ e independentes da cadeia de Markov $\{\theta(n) ; n \geq 0\} . w_{n}$ e $x_{n}$ são vetores aleatórios independentes. $\xi_{n}^{\ell} \in \mathbb{R}^{n_{\xi}}$ é uma parâmetro determinístico variante no tempo que pertence ao simplex unitário:

$\Xi=\left\{\xi_{n} \in \mathbb{R}^{n_{\xi}} ; \xi_{n}^{\ell} \geq 0, \sum_{\ell=1}^{n_{\xi}} \xi_{n}^{\ell}=1, \ell=1, \ldots, n_{\xi}\right\}$.

Para cada $\theta_{n}=i \in \Theta$, as matrizes $A_{\xi_{n}}(i)$ e $B_{\xi_{n}}(i)$ dependem de uma maneira afim dos parâmetros variantes no tempo $\xi_{n} \in \Xi$ como segue:

$$
A_{\xi_{n}}(i)=\sum_{\ell=1}^{n_{\xi}} \xi_{n}^{\ell} A_{\ell}(i) \text { e } B_{\xi_{n}}(i)=\sum_{\ell=1}^{n_{\xi}} \xi_{n}^{\ell} B_{\ell}(i) .
$$

Para $\ell=1, \ldots, n_{\xi}$ e para todo $i \in \Theta$, as matrizes $A_{\ell}(i) \in \mathbb{R}^{n_{x} \times n_{x}}$ e $B_{\ell}(i) \in \mathbb{R}^{n_{x} \times n_{u}}$ são dadas. Também são dadas as matrizes $E(i) \in \mathbb{R}^{n_{x} \times n_{w}}, C(i) \in \mathbb{R}^{n_{x} \times n_{y}}$ e $D(i) \in \mathbb{R}^{n_{x} \times n_{u}}$ para todo $i \in \Theta$.
O critério de custo linear quadrático de horizonte finito associado ao sistema (1), para uma lei de controle admissível $u=\left(u_{0}, \ldots, u_{N-1}\right)$, é dado por:

$$
J_{N}(u)=\sum_{n=0}^{N-1} \mathbb{E}\left[\left\|y_{n}\right\|^{2}\right]+\mathbb{E}\left[x_{N}^{\prime} \mathcal{Q}\left(\theta_{N}\right) x_{N}\right],
$$

em que $N<\infty$ é o horizonte de tempo, as matrizes $\mathcal{Q}\left(\theta_{N}\right) \in \mathcal{S}^{n 0}, C^{\prime}\left(\theta_{n}\right) C\left(\theta_{n}\right) \in \mathcal{S}^{n 0}$ e $D^{\prime}\left(\theta_{n}\right) D\left(\theta_{n}\right) \in \mathcal{S}^{n+}$, $n=0, \ldots, N-1$ representam as matrizes de ponderações $\operatorname{com} C^{\prime}\left(\theta_{n}\right) D\left(\theta_{n}\right)=0, n=0, \ldots, N-1$.

Para o projeto de controle, a lei escolhida é da forma usual de realimentação de estados dependente dos modos de Markov como segue:

$$
u_{n}=G_{n}\left(\theta_{n}\right) x_{n}, \quad 0 \leq n \leq N-1 .
$$

Neste trabalho, deseja-se determinar os ganhos $G_{0}(i), \ldots$, $G_{N-1}(i), i=1, \ldots, n_{\theta}$ em (3) de modo a minimizar o funcional em (2). Em termos formais, deseja-se obter $u^{*}$ tal que:

$$
\begin{gathered}
u^{*}=\underset{u}{\arg \min } J_{N}(u) \\
\text { sujeito a (1) e (3). }
\end{gathered}
$$

A novidade na abordagem proposta neste artigo quando comparada com a solução ótima de Riccati está no fato de que (4) considera robustez que depende de conjuntos politópicos variantes no tempo. O problema em (4) está ainda aberto na literatura, porque para este não existe solução algébrica. Neste trabalho, desenvolve-se condições por meio de desigualdades matriciais lineares e obtémse um controle subótimo $\bar{u}=\left\{\bar{u}_{0}, \ldots, \bar{u}_{N-1}\right\}$ tal que $J_{n}\left(u^{*}\right) \leq J_{n}(\bar{u}), n=0, \ldots, N-1$.

Para facilitar a notação, para toda sequência $M \in \mathbb{S}^{n+}$ define-se o operador $\mathcal{E}(M)=\left(\mathcal{E}_{1}(M), \ldots, \mathcal{E}_{n_{\theta}}(M)\right)$ como:

$$
\mathcal{E}_{i}(M):=\sum_{k=1}^{n_{\theta}} p(i, j) M(j)
$$

\section{RESULTADOS PRELIMINARES}

Suponha que no $q$-ésimo estágio, sejam conhecidos $x(q)=$ $x \in \mathbb{R}^{n_{x}}$ e $\theta(q)=i \in \Theta$. Então, o "cost-to-go" associado ao funcional (2) é definido por (Bertsekas, 1976, p. 65):

$$
\begin{aligned}
J_{N, q}(u)= & \sum_{n=q}^{N-1} \mathbb{E}\left[x_{n}^{\prime} C^{\prime}\left(\theta_{n}\right) C\left(\theta_{n}\right) x_{n}+u_{n}^{\prime} D_{n}^{\prime}\left(\theta_{n}\right) D_{n}\left(\theta_{q}\right) u_{n}\right. \\
& \left.+x_{N} \mathcal{Q}(\theta(N)) x_{N} \mid x_{q}=x, \theta_{q}=i\right]
\end{aligned}
$$

Note de $(2)$ e $(6)$ que $J_{N}(u)=J_{N, 0}(u)$.

Percebe-se que ao substituir (3) em (6), o custo em (6) torna-se idêntico a

$$
\begin{aligned}
J_{N, q}(u)= & \sum_{n=q}^{N-1} \mathbb{E}\left[x_{n}^{\prime} C^{\prime}\left(\theta_{n}\right) C\left(\theta_{n}\right) x_{n}\right. \\
& +x_{n}^{\prime} G_{n}^{\prime}\left(\theta_{n}\right) D_{n}^{\prime}\left(\theta_{n}\right) D_{n}\left(\theta_{q}\right) G_{n}\left(\theta_{n}\right) x_{n} \\
& \left.+x_{N} \mathcal{Q}(\theta(N)) x_{N} \mid x_{q}=x, \theta_{q}=i\right] .
\end{aligned}
$$

A utilidade do "cost-to-go" em (6) ficará clara no resultado que segue em breve. Antes define-se a seguir funções 
auxiliares $V_{n}: \mathbb{R}^{n_{x}} \times \Theta \rightarrow \mathbb{R}_{+}, n=0, \ldots, N$, que serão úteis na avaliação do custo em (7).

Seja $\bar{A}_{n}(i)=A_{\xi_{n}}(i)+B_{\xi_{n}}(i) G_{n}(i)$.

Considere:

$$
\mathcal{V}_{n}\left(x_{n}, \theta_{n}\right)=x_{n}^{\prime} P_{n}\left(\theta_{n}\right) x_{n}+\alpha_{n}\left(\theta_{n}\right), \quad n=0, \ldots, N,
$$

para $G_{n}(i), i=1, \ldots, n_{\theta}, n=0, \ldots, N-1$ satisfazendo

(3) matrizes $P_{n}(i)$ que devem satisfazer a recorrência:

$$
\begin{aligned}
P_{n}(i)= & C^{\prime}(i) C(i)+G_{n}^{\prime}(i) D^{\prime}(i) D(i) G_{n}(i) \\
& +\bar{A}_{n}^{\prime}(i) \mathcal{E}_{i}\left(P_{n+1}\right) \bar{A}_{n}(i), n=0, \ldots, N-1, \\
\alpha_{n}(i)= & \mathcal{E}_{i}\left(\alpha_{n+1}\right)+ \\
& \operatorname{Tr}\left\{E^{\prime}(i) \mathcal{E}_{i}\left(P_{n+1}\right) E(i) \Sigma\right\} n=0, \ldots, N-1, \\
\alpha_{N}(i)= & 0, P_{N}(i)=\mathcal{Q}(i), i=1, \ldots, n_{\theta} .
\end{aligned}
$$

Lema 1. Seja $q \in\{0, \ldots, N\}$ fixado. Assuma que o par $\left(\theta_{q}, x_{q}\right)$ é conhecido. Então é válida a seguinte igualdade

$$
J_{N, q}(u)=\mathcal{V}_{q}\left(x_{q}, \theta_{q}\right) .
$$

A demonstração do Lema 1 está disponível em [Proposição 5, (Vargas et al., 2005)].

O próximo resultado mostra um modo de avaliar um limitante superior para a função de custo intermediário em (6) quando o seguinte problema é factível.

Problema 2. Seja $q \in\{0, \ldots, N-1\}$ fixado. Sejam $\theta_{q} \in \Theta$, $x_{q} \in \mathbb{R}^{n_{x}}$ e os ganhos $G_{q}(i), \ldots, G_{N-1}(i) \in \mathbb{R}^{n_{u} \times n_{x}}$ dados. Sejam $\hat{P}_{n}(i) \in \mathcal{S}^{n+}, \hat{\alpha}_{n}(i) \in \mathcal{S}^{+}$variáveis; $i=1, \ldots, n_{\theta}$ e $n=q, \ldots, N-1$. Encontrar $\gamma_{q} \geq 0$ tal que:

$$
\begin{aligned}
\gamma_{q}>x_{q}^{\prime} & \hat{P}_{q}\left(\theta_{q}\right) x(q)+\hat{\alpha}_{q}\left(\theta_{q}\right), \\
\hat{P}_{n}(i)> & C^{\prime}(i) C(i)+G_{n}^{\prime}(i) D^{\prime}(i) D(i) G_{n}(i) \\
& +\bar{A}_{n}^{\prime}(i) \mathcal{E}_{i}\left(\hat{P}_{n+1}\right) \bar{A}_{n}(i), n=q, \ldots, N-1, \\
\hat{\alpha}_{n}(i)> & \mathcal{E}_{i}\left(\hat{\alpha}_{n+1}\right) \\
& +\operatorname{Tr}\left\{E^{\prime}(i) \mathcal{E}_{i}\left(\hat{P}_{n+1}\right) E(i) \Sigma\right\}, n=q, \ldots, N-1, \\
\hat{P}_{N}(i)= & \mathcal{Q}(i), \hat{\alpha}_{N}(i)=0, i=1, \ldots, n_{\theta} .
\end{aligned}
$$

O próximo resultado será útil para dar suporte em como solucionar o 2, sendo sua demonstração imediata.

Lema 3. Sejam $\theta_{q} \in \Theta$ e $x_{q} \in \mathbb{R}^{n_{x}}$ dados para algum $q \in\{0, \ldots, n-1\}$. Então,

$$
\gamma_{q}>\mathcal{V}_{q}\left(x_{q}, \theta_{q}\right)=J_{N, q}(u)
$$

\section{RESULTADO PRINCIPAL}

O próximo teorema, apresenta a solução para o Problema 2.

Teorema 4. Sejam $K_{0}(i), \ldots, K_{N-1}(i) \in \mathbb{R}^{n_{u} \times n_{x}}$, ganhos quaisquer dados, para todo $i \in \Theta$. Se existirem matrizes $P_{n}(i) \in \mathbb{S}^{n_{x}^{+}}, F_{n}(i) \in \mathbb{R}^{n_{x} \times n_{x}}, H_{n}(i) \in \mathbb{R}^{n_{y} \times n_{y}}$, para todo $i \in \Theta$ e para $n=1, \ldots, N$, matrizes $L_{n}(i) \in \mathbb{R}^{n_{u} \times n_{x}}$, $R_{n}(i) \in \mathbb{R}^{n_{x} \times n_{x}}$ para $n=1, \ldots, N-1$ e existir um escalar $\rho$ de forma que:

$$
x_{0}^{\prime} \hat{P}_{0}(i) x_{0}+\alpha_{0}(i)<\rho,
$$

$$
\begin{aligned}
& \hat{\alpha}_{n}(i)>\mathcal{E}_{i}\left(\hat{\alpha}_{n+1}\right)+\operatorname{Tr}\left\{E^{\prime}(i) \mathcal{E}_{i}\left(\hat{P}_{n+1}\right) E(i) \Sigma\right\} \\
& \hat{\alpha}_{N}(i)=0 .
\end{aligned}
$$

e tal que a LMI (13) (descrita em separado ao longo do artigo) seja factível, então $G_{n}(i)=R_{n}^{-1}(i) L_{n}(i)$ é um ganho de controle dependente do modo. Além disso,

$$
J_{N}\left(u^{*}\right)<\rho \text {. }
$$

Demonstração. Suponha (13) factível e multiplicando-a por

$$
\left[\begin{array}{cccc}
I & 0 & 0 & \left(R_{n}^{-1}(i) L_{n}(i)-K_{n}(i)\right)^{\prime} \\
0 & I & 0 & 0 \\
0 & 0 & I & 0
\end{array}\right]
$$

pela esquerda e por sua transporta à direita, obtém-se a equação (15) (descrita em separado ao longo do artigo). Agora, multiplicando (15) por

$\left[\begin{array}{ll}I & A_{\ell}^{\prime}(i)+B_{\ell}(i) R_{n}^{-1}(i) L_{n}(i) \quad C(i)+D^{\prime}(i) R_{n}^{-1}(i) L_{n}(i)\end{array}\right]$ pela esquerda e por sua transposta à direita, obtém-se:

$$
\begin{aligned}
\hat{P}_{n}(i)> & \left(A_{\ell}(i)+B_{\ell}(i) G_{n}(i)\right)^{\prime} \mathcal{E}_{i}\left(\hat{P}_{n+1}\right)\left(A_{\ell}(i)+B_{\ell}(i) G_{n}(i)\right) \\
& +C^{\prime}(i) C(i)+\left(D(i) G_{n}(i)\right)^{\prime}\left(D(i) G_{n}(i)\right)
\end{aligned}
$$

em que $G_{n}(i)=R_{n}^{-1}(i) L_{n}(i)$.

Note que as desigualdades (11), (12) e (16) satisfazem o Problema 2. Portanto, utilizando o resultado do Lema 3 a demonstração está completa.

\section{EXEMPLO NUMÉRICO}

Nesta seção, o resultado apresentado no Teorema 4 é ilustrado analisando um sistema linear com saltos Markovianos discreto no tempo com dois modos de operação, para os parâmetros fixados:

Para o modo $i=1$, as matrizes do sistema são dadas por:

$$
\begin{gathered}
A_{1}(1)=\left[\begin{array}{cc}
-0.9 & 0.3 \\
0.1 & -0.3
\end{array}\right], A_{2}(1)=\left[\begin{array}{cc}
1.3 & -1.8 \\
-0.4 & -0.5
\end{array}\right], \\
B_{1}(1)=\left[\begin{array}{c}
-1.9 \\
0.8
\end{array}\right], B_{2}(1)=\left[\begin{array}{c}
1.1 \\
-1.3
\end{array}\right], E(1)=\left[\begin{array}{ll}
1 & 0 \\
0 & 1
\end{array}\right], \\
C(1)=\left[\begin{array}{cc}
0.01 & 0 \\
0 & 0.01 \\
0 & 0
\end{array}\right], D(1)=\left[\begin{array}{lll}
0 & 0 & 0.01
\end{array}\right] .
\end{gathered}
$$

Para o modo $i=2$, as matrizes do sistema são:

$$
\begin{gathered}
A_{1}(2)=\left[\begin{array}{cc}
0 & -1.7 \\
-0.5 & -0.6
\end{array}\right], A_{2}(2)=\left[\begin{array}{cc}
1.6 & -0.7 \\
-0.7 & -1.2
\end{array}\right], \\
B_{1}(2)=\left[\begin{array}{l}
-1.1 \\
-1.2
\end{array}\right], B_{2}(2)=\left[\begin{array}{c}
1.1 \\
-1.7
\end{array}\right], E(2)=\left[\begin{array}{ll}
1 & 0 \\
0 & 1
\end{array}\right], \\
C(2)=\left[\begin{array}{cc}
0.01 & 0 \\
0 & 0.01 \\
0 & 0
\end{array}\right], D(2)=\left[\begin{array}{lll}
0 & 0 & 0.01
\end{array}\right] .
\end{gathered}
$$

A matriz de probabilidade de transição e a matriz de covariância são dadas, respectivamente, por:

$$
\mathbb{P}=\left[\begin{array}{ll}
0.99 & 0.01 \\
0.02 & 0.98
\end{array}\right], \Sigma=\left[\begin{array}{cc}
0.1 & 0 \\
0 & 0.1
\end{array}\right]
$$




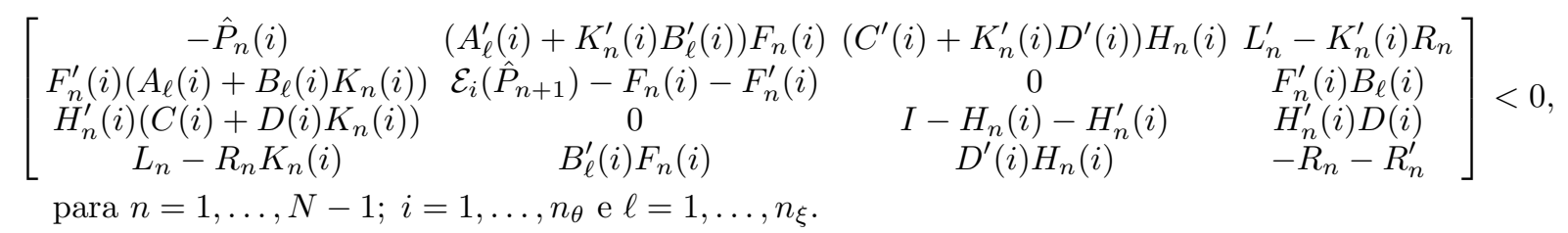

$$
\begin{gathered}
{\left[\begin{array}{ccc}
-\hat{P}_{n}(i) & \left(A_{\ell}^{\prime}(i)+B_{\ell}(i) R_{n}^{-1} L_{n}\right)^{\prime} F_{n}(i) & \left(C(i)+D^{\prime}(i) R_{n}^{-1} L_{n}\right)^{\prime} H_{n}(i) \\
F_{n}\left(A_{\ell}(i)+B_{\ell}(i) R_{n}^{-1} L_{n}\right) & \mathcal{E}(i)\left(\hat{P}_{n+1}\right)-F_{n}(i)-F_{n}^{\prime}(i) & 0 \\
H_{n}^{\prime}(i)\left(C(i)+D(i) R_{n}^{-1} L_{n}\right) & 0 & I-H_{n}(i)-H_{n}^{\prime}(i)
\end{array}\right]<0,} \\
\text { para } n=1, \ldots, N-1 ; i=1, \ldots, n_{\theta} \text { e } \ell=1, \ldots, n_{\xi} .
\end{gathered}
$$

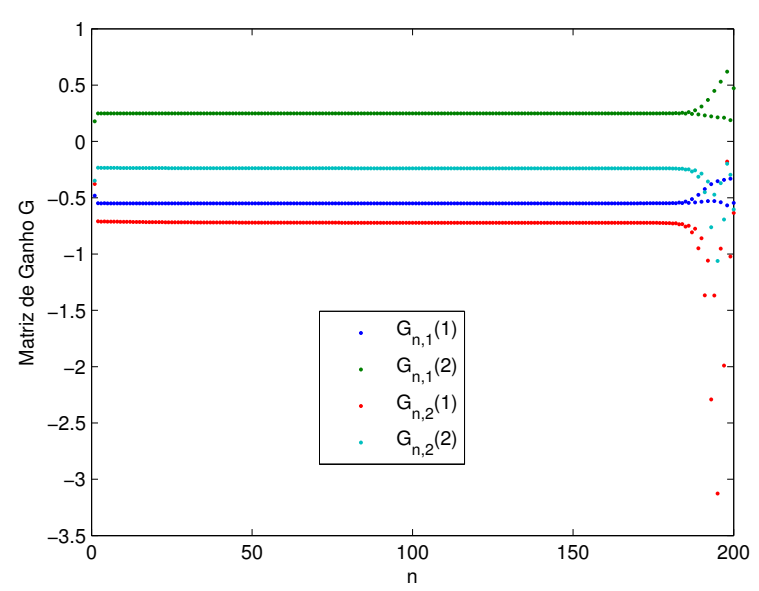

Figura 1. Ganhos de realimentação de estados obtidos para $N=200 . G_{n, 1}=G_{n, 1}(\theta(n))$ e $G_{n, 2}=G_{n, 2}(\theta(n))$.

O Teorema 4 exige que sejam dados os ganhos $K_{1}(1), \ldots$, $K_{N}(1), K_{1}(2), \ldots, K_{N}(2)$. Neste exemplo em particular, foram fixados os ganhos idênticos aos ganhos de Riccati que podem ser encontrados em [Seção 2.4.1, pag. 26 , (Costa et al., 2006)]. Tais ganhos foram aplicados nas LMIs (11), (12) e (13) com $N=200$. As curvas obtidas foram mostradas na Figura 1.

Observe que para a maioria dos estágios $n$, os ganhos $G_{n, 1}(i)$ e $G_{n, 2}(i)$ tendem aos valores

$$
\begin{aligned}
& G_{n, 1}(1) \simeq-0.54, G_{n, 2}(1) \simeq-0.72, \\
& G_{n, 1}(2) \simeq 0.24, G_{n, 2}(2) \simeq-0.23 .
\end{aligned}
$$

Especula-se que o método LMI pode ser capaz de gerar ganhos estacionários porém, investigação adicional é necessária.

Além disso, o valor obtido para $\rho$, que é o limitante superior para o custo linear quadrático de horizonte finito $J_{N}\left(u^{*}\right)$, é dado por 0.918441 .

\section{CONCLUSÃO}

Este trabalho apresentou uma solução subótima para um problema de controle linear quadrático de tempo finito de sistemas lineares com saltos Markovianos, com parâmetros variantes no tempo e ruído aditivo. Ganhos de realimentação dependentes do modo de Markov e do tempo são calculados resolvendo um problema de otimização por meio de desigualdades matriciais lineares dadas pelo Teorema 4. Um simulação numérica foi mostrada para ilustrar a efetividade do método proposto.

\section{AGRADECIMENTOS}

Esse trabalho foi financiado por CAPES, CNPq e FAPEMIG.

\section{REFERÊNCIAS}

Bertsekas, D.P. (1976). Dynamic programing and stochastic control. Mathematics in science and engineering, 125.

Costa, O.L.V., Boukas, E.K., and Marques, R.P. (1999). Constrained quadratic control of discrete-time Markovian jump linear systems. Automatica, 35(4), 617-626.

Costa, O.L.V., Fragoso, M.D., and Marques, R.P. (2006). Discrete-time Markov jump linear systems. Springer Science \& Business Media.

Costa, O.L.V., Fragoso, M., and Todorov, M.G. (2014). A new approach for the $\mathrm{H}_{2}$ control of Markov jump linear systems with partial information. IFAC Proceedings Volumes, 47(3), 11099-11104.

Costa, O.L.V. and Tuesta, E.F. (2003). Finite horizon quadratic optimal control and a separation principle for Markovian jump linear systems. IEEE Transactions on Automatic Control, 48(10), 1836-1842.

Do Val, J.B., Geromel, J.C., and Gonçalves, A.P. (2002). The $\mathrm{H}_{2}$-control for jump linear systems: cluster observations of the Markov state. Automatica, 38(2), 343-349.

Gagliardi, G., Casavola, A., and Famularo, D. (2012). A fault detection and isolation filter design method for Markov jump linear parameter-varying systems. International Journal of Adaptive Control and Signal Processing, 26(3), 241-257.

Geromel, J.C., Gonçalves, A.P., and Fioravanti, A.R. (2009). Dynamic output feedback control of discretetime Markov jump linear systems through linear matrix inequalities. SIAM Journal on Control and Optimization, 48(2), 573-593.

Kim, S.H. (2017). $\mathrm{H}_{2}$ control of Markovian jump LPV systems with measurement noises: Application to a DCmotor device with voltage fluctuations. Journal of the Franklin Institute, 354(4), 1784-1800.

Lopes, R.O., Mendes, E.M.A.M., Torres, L.A.B., and Palhares, R.M. (2019). Constrained robust model predicted control of discrete-time Markov jump linear systems. IET Control Theory \& Applications, 13(4), 517-525. 
Mariton, M. (1990). Jump Linear Systems in Automatic Control. Marcel Dekker, New York.

Morais, C.F., Palma, J.M., Peres, P.L., and Oliveira, R.C. (2018). An lmi approach for reduced-order filtering of uncertain discrete-time Markov and bernoulli jump linear systems. Automatica, 95, 463-471.

Oliveira, R.C., Vargas, A.N., do Val, J.B., and Peres, P.L. (2014). Mode-independent $\mathrm{H}_{2}$-control of a DC motor modeled as a Markov jump linear system. IEEE Transactions on Control Systems Technology, 22(5), 1915-1919.

Ren, H. and Zong, G. (2017). Asynchronous input-output finite-time filtering for switched LPV systems. Journal of the Franklin Institute, 354(14), 6292-6311.

Shi, P. and Li, F. (2015). A survey on Markovian jump systems: modeling and design. International Journal of Control, Automation and Systems, 13(1), 1-16.

Shi, P., Boukas, E.K., and Agarwal, R.K. (1999). Control of Markovian jump discrete-time systems with norm bounded uncertainty and unknown delay. IEEE Transactions on Automatic Control, 44(11), 2139-2144.

Vargas, A.N., Costa, E.F., and do Val, J.B. (2013). On the control of Markov jump linear systems with no mode observation: application to a DC motor device. International Journal of Robust and Nonlinear Control, 23(10), 1136-1150.

Vargas, A.N., do Val, J.B., and Costa, E.F. (2005). Controle de horizonte retrocedente de sistemas lineares com saltos Markovianos para o problema de rastreamento com alvos dinâmicos. Sba: Controle \& Automação Sociedade Brasileira de Automatica, 16(4), 435-448.

Zhu, Y., Zhong, Z., Zheng, W.X., and Zhou, D. (2017). HMM-based $\mathrm{H}_{\infty}$ filtering for discrete-time Markov jump LPV systems over unreliable communication channels. IEEE Transactions on Systems, Man, and Cybernetics: Systems, (99), 1-12. 